\title{
A moving boundary problem for the Stokes equations involving osmosis: Variational modelling and short-time well-posedness
}

\author{
FRIEDRICH LIPPOTH ${ }^{1}$, MARK A. PELETIER ${ }^{2}$ and \\ GEORG PROKERT ${ }^{2}$ \\ ${ }^{1}$ Institute of Applied Mathematics, Leibniz University Hannover, Welfengarten 1, D-30167 Hannover, Germany \\ email: lippoth@ifam.uni-hannover.de \\ ${ }^{2}$ Faculty of Mathematics and Computer Science, TU Eindhoven, P.O. Box 513, \\ $5600 \mathrm{MB}$ Eindhoven, the Netherlands \\ emails: m.a.peletier@tue.nl,g.prokert@tue.nl
}

(Received 9 April 2014; revised 12 October 2015; accepted 15 October 2015; first published online 24 November 2015)

\begin{abstract}
Within the framework of variational modelling we derive a one-phase moving boundary problem describing the motion of a semipermeable membrane enclosing a viscous liquid, driven by osmotic pressure and surface tension of the membrane. For this problem we prove the existence of classical solutions for a short-time.
\end{abstract}

Key words: variational modelling, Stokes equations, osmosis, moving boundary problem, maximal continuous regularity

\section{Introduction}

It is the aim of the present paper to introduce and discuss a model for the motion of a closed membrane in a liquid, taking into account simultaneously the following effects:

(1) surface tension forces of the membrane,

(2) diffusion of a solute in the liquid,

(3) (quasistationary) viscous motion of the liquid,

(4) osmotic pressure difference across the membrane,

(5) resistance to liquid motion through the membrane.

To avoid additional difficulties, our attention in this paper is restricted to a one-phase problem, i.e. we assume that the liquid outside the membrane has negligible viscosity and does not contain any solute.

We remark that quite a number of well-studied moving boundary problems are contained as special or limit cases in the above general setting. For example, if (1) and (3) are the only forces taken into account and the membrane is impermeable, so-called quasistationary Stokes flow (driven by surface tension) arises. If only (1), (2), and (4) are considered, an osmosis model without liquid motion occurs. In the 
limit of fast diffusion, this problem (formally) yields a mean curvature flow problem with a non-local lower order term. For details and references on this, we refer to Section 2.

It is reasonable to demand that a model for the full problem should recover these special and limiting cases. The crucial observation here is that both the osmosis problem and the Stokes flow problem have a variational structure, i.e. they can be interpreted as gradient flows with respect to certain energies and dissipation functionals. This yields a straightforward approach to the full problem: We use linear combinations for both energy and dissipation and assume that the evolution we are interested in is a generalised gradient flow with respect to these. More precisely, an additional dissipation term is introduced to model (finite) resistance of the membrane against solute flux.

We will use this approach to formally derive a moving boundary PDE system that describes our full problem. At the moment it seems quite challenging to use its variational structure for deriving existence results under weak smoothness assumptions. Therefore, we do not pursue this approach here. Instead, we show short-time well-posedness of the PDE system in a classical setting by transformation to a fixed domain and applying maximalregularity results and a contraction argument to the resulting non-linear parabolic Cauchy problem in a product of suitable function spaces, in a fashion oriented at [13].

This paper is organised as follows: In Section 2, we introduce the model and discuss several limit cases. In Section 3.1, we collect some necessary technical material such as a regularity result in the scale of little Hölder spaces for the Stokes equations. We define the notion of a classical solution of the full problem and state our main result (Theorem 3.3). Section 3.2 contains its proof.

\section{Modelling and limit cases}

\subsection{Variational modelling: basic approach and preliminary examples}

The modelling philosophy that we use here is a generalisation of a well-known strategy for stationary problems. When deriving equations for stationary problems, for instance for elasticity equations, it is common to postulate an energy and assume that the "system" minimises this energy, possibly under constraints. The stationarity condition that follows typically has the form of a differential equation.

Generalisations of this principle to non-stationary problems go back at least to Rayleigh [20]. In such generalisations, both an energy and a dissipation mechanism are postulated, and the system is assumed to follow the path given by "incremental" minimisation. We now know that all gradient flows can be obtained in this way, and in addition, by postulating the driving functional ("energy") and the dissipation mechanism, the system is completely characterised.

This implies that it is possible to derive the equations of motion in a given gradient-flow system by choosing an energy and a dissipation mechanism. The lecture notes [19] give an extensive introduction to this method of modelling. It is an advantage of this modelling approach that in many applications it results in modelling choices that are independent from each other, relatively straightforward, and can be justified from physical principles. By contrast, the resulting systems of differential equations might be less transparent. 


\subsubsection{The general setting}

Defining a variational model comprises the following choices:

- a set $\mathscr{Z}$ of states with the structure of a differentiable (possibly infinite-dimensional) manifold (the state manifold);

- a differentiable energy functional $\mathscr{E}: \mathscr{Z} \longrightarrow \mathbb{R}$;

- a process space $\mathscr{P}_{z}$ at all $z \in \mathscr{Z}$; (In fact, it makes sense to consider $\mathscr{P}:=\bigcup_{z \in \mathscr{Z}} \mathscr{P}_{z}$ as a vector bundle over $\mathscr{Z}$.)

- a dissipation functional $\Psi_{z}: \mathscr{P}_{z} \longrightarrow \mathbb{R}$,

- a linear (bundle) map $\Pi_{z}: \mathscr{P}_{z} \longrightarrow T_{z} \mathscr{Z}$, where $T_{z} \mathscr{Z}$ denotes the tangent space to $\mathscr{Z}$ at $z$. We will refer to $\Pi_{z}$ as process map.

(These are formal, i.e. we will assume sufficient smoothness and do not strictly define the phase manifold, its tangent space, etc.)

With these choices, the model is given as a dynamical system on $\mathscr{Z}$ defined by

$$
\dot{z}=\Pi_{z} w^{*}
$$

where $w^{*}$ is the solution to the minimisation problem

$$
\Psi_{z}(w)+\mathscr{E}^{\prime}(z)\left[\Pi_{z} w\right] \longrightarrow \min , \quad w \in \mathscr{P}_{z}
$$

Here, $\mathscr{E}^{\prime}(z)[s]$ is the Fréchet derivative of $\mathscr{E}$ at $z$ applied to the tangent vector $s$. The minimiser $w^{*}$ can be considered as the "actual process chosen by the system in state $z$ " in the trade-off between diminishing the energy and minimising dissipation at $z \in \mathscr{Z}$. We assume here that the minimisation problem (2.2) is uniquely solvable, a property that hinges on strict convexity and coercivity in appropriate norms of the potential $\Psi_{z}$. In various other applications, such as the rate-independent systems that appear in fracture, plasticity, and hysteresis [18], these properties fail; we refrain from giving details on this as our interest is restricted to a situation in which the above framework is sufficient.

Observe, furthermore, that the approach described here contains the concept of a gradient flow as a special case, as mentioned above: If $(\mathscr{Z}, g)$ is a Riemannian manifold, $\mathscr{P}_{z}=T_{z} \mathscr{Z}, \Psi_{z}(w)=\frac{1}{2} g_{z}(w, w)$, and $\Pi_{z}$ is the identity, then (2.1) defines the gradient flow on $(\mathscr{Z}, g)$ induced by $\mathscr{E}$. On the other hand, if the dissipation functional $\Psi_{z}$ is quadratic (i.e. $\left.\Psi_{z}(w)=\Phi_{z}(w, w)\right)$ and positive definite, and if the process map is surjective, any evolution of type (2.1), (2.2) can be considered as a gradient flow with respect to $\mathscr{E}$ and the Riemannian metric $g_{z}$ constructed as follows: let $Q_{z}$ be the $\Psi_{z}$-orthogonal projection along $\operatorname{ker} \Pi_{z}$. Observe that $Q_{z}$ is constant along fibres $\Pi_{z}^{-1}(v), v \in T_{z} \mathscr{Z}$. Using this, it is straight forward to check that the bilinear map

$$
g_{z}\left(v_{1}, v_{2}\right):=\Phi_{z}\left(Q_{z} w_{1}, Q_{z} w_{2}\right), \quad \Pi_{z} w_{i}=v_{i}(i=1,2)
$$

is a well-defined Riemannian metric on $T_{z} \mathscr{Z}$ that has the desired property.

Before we describe the model we are interested in, we will informally illustrate the approach of variational modelling in two related but simpler, paradigmatic and 
well-understood problems. This will also provide a motivation for the choices we are going to make for the osmosis model.

\subsubsection{Diffusion [19, Section 5.5]}

To model linear diffusion on $\mathbb{R}^{N}$, the following choices are possible within the above framework. $\mathscr{Z}$ is a suitable set of scalar non-negative functions on $\mathbb{R}^{N}$ representing concentrations. The functional $\mathscr{E}$ is given by the entropy

$$
\mathscr{E}(c):=\gamma \int_{\mathbb{R}^{N}} c \ln c d x, \quad \gamma>0 \text { fixed, } c \in \mathscr{Z} .
$$

(We remark that $c$ is understood to be dimensionless, i.e. a ratio with respect to a fixed reference concentration $c^{0}$. For simple, approximately spherical particles, we have $\gamma=R T c^{0}$, where $R$ is the universal gas constant, $T$ is absolute temperature, and $c^{0}$ is the same normalisation concentration, see e.g. [19, Ch. 4]). The process space consists of vector fields on $\mathbb{R}^{N}$ representing the mass flux, with dissipation functional

$$
\Psi_{c}(f):=\frac{\eta_{1}}{2} \int_{\mathbb{R}^{N}} \frac{|f|^{2}}{c} d x, \quad f \in \mathscr{P}_{c} .
$$

(This is $+\infty$ whenever $c=0$ and $f \neq 0$ on a set of non-zero measure, so for the actual process, $f=0$ is enforced where $c=0$.) The constant $\eta_{1}$ in this expression is an inverse mobility of the solute. For dilute, approximately spherical solute particles it should be taken equal to $6 \pi r v$, where $r$ is the particle radius, and $v$ the dynamic viscosity of the solvent [19, Ch. 5].

Naturally, the tangent space $T_{c}(\mathscr{Z})$ has to be interpreted as the set of the "local concentration changes". Accordingly, to encode mass conservation we choose

$$
\Pi_{c} f:=-\operatorname{div} f, \quad f \in \mathscr{P}_{c} .
$$

Solving (2.2) with these choices yields $\eta_{1} f^{*}=\gamma \nabla c$ and from (2.1) we get

$$
c_{t}=\frac{\gamma}{\eta_{1}} \Delta c \quad \text { on } \mathbb{R}^{N} .
$$

\subsubsection{Free-boundary Stokes flow driven by curvature}

To describe the motion of a liquid drop that is deformed by surface tension forces via "creeping flow", it is natural to choose $\mathscr{Z}$ to be the (infinite-dimensional) manifold of (simply connected) domains $\Omega$, representing the drop shapes. The energy is the corresponding surface measure:

$$
\mathscr{E}(\Omega):=\alpha|\partial \Omega|=\alpha \int_{\partial \Omega} d \sigma,
$$

where $\alpha>0$ is the surface energy density. The tangent space $T_{\Omega} \mathscr{Z}$ can be represented by functions $V_{n}: \partial \Omega \longrightarrow \mathbb{R}$ that play the role of the normal velocities of the boundary of a moving domain $t \mapsto \Omega(t)$. 
Again it is straightforward to choose a suitable process space, namely, the space of all divergencefree vector fields in $\Omega$, with dissipation caused by inner friction of the liquid:

$$
\Psi_{\Omega}(v):=\frac{\eta_{2}}{2} \int_{\Omega}|\varepsilon(v)|^{2} d x, \quad \varepsilon(v)=\frac{1}{2}\left(\nabla v+(\nabla v)^{T}\right), \quad v \in \mathscr{P}_{\Omega} .
$$

These choices are implied by the assumptions that the liquid is Newtonian and incompressible, and that its mass is preserved. The constant $\eta_{2}$ is the shear viscosity. The mapping $\Pi_{\Omega}$ is chosen to represent the kinematic boundary condition, i.e. the assumption that the boundary of the drop moves along with the liquid particles that constitute it:

$$
\Pi_{\Omega}(v):=\left.v\right|_{\partial \Omega} \cdot n
$$

where $n$ is the exterior unit normal to $\Omega$.

A straightforward calculation shows that (2.1), (2.2) now yield the moving boundary problem

$$
V_{n}=u \cdot n \quad \text { on } \partial \Omega(t)
$$

where $u$ solves the Stokes system

$$
\begin{aligned}
& \frac{\eta_{2}}{2} \Delta u-\nabla p=0 \quad \text { in } \Omega(t), \quad t>0, \\
& \operatorname{div} u=0 \quad \text { in } \Omega(t), \quad t>0, \\
& \tau(u, p) n=\alpha H n \text { on } \partial \Omega(t), t>0 \text {, }
\end{aligned}
$$

where $p$ is the hydrodynamic pressure, occurring here as a Lagrange multiplier corresponding to the incompressibility condition, $H$ is the sum of the principal curvatures of $\partial \Omega$, and

$$
\tau(u, p)=\eta_{2} \varepsilon(u)-p I,
$$

is the hydrodynamic stress tensor. We have to remark here that the Stokes system defines $u$ only up to rigid body velocities since these are in the kernel of $\varepsilon$, see below. This moving boundary problem (as well as closely related ones) has been discussed extensively in the literature, see e.g. $[2,7,10,11,22]$.

In both problems discussed here, the following observations can be made:

- Quadratic dissipation functionals correspond to linear constitutive relations (Fick's law and Newtonian stress-strain relation, respectively).

- In these cases and many others, the maps $\Pi_{z}$ encode balance laws (mass balance in the first example, "boundary conservation" in the second).

\subsection{Variational modelling of the Stokes-Osmosis problem}

In the process of variational modelling, the above examples will play a guiding role. We will need to deal with some additional aspects: mass conservation of the solvent in the presence of a moving boundary, dissipation by the osmotic process, and diffusion in a moving solvent.

We start by listing our modelling assumptions. 
- The solvent is incompressible and moves with velocity field $u$. Mass conservation of the solvent then implies

$$
\operatorname{div} u=0 \quad \text { in } \Omega .
$$

- The solute moves according to a flux field $f$ so that mass conservation of the solute is expressed by

$$
c_{t}+\operatorname{div} f=0 \quad \text { in } \Omega .
$$

- The membrane is impermeable to the solute. Together with mass conservation this implies the boundary condition

$$
f \cdot n=c V_{n} \quad \text { on } \partial \Omega .
$$

- The solvent inside the cell is viscous with a linear dependence of the strain rate on the stress.

- The solute motion is governed by convection along $u$ and diffusion through the solvent. The diffusion obeys Fick's law.

- There is finite resistance by the membrane to solvent moving through it, proportional to the solvent flux.

These assumptions obviously oversimplify the physics of any "real" membrane enclosing a liquid in motion. No further mechanical properties except resistance to area growth from normal displacement are taken into account; in particular, there is no resistance to tangential stretching (or one has to assume completely frictionless slipping of the liquid in tangential direction along the membrane). Including these effects, in the vein of e.g. [5] seems to be interesting but highly non-trivial and has to remain outside the scope of this paper.

Our model is encoded in the following choices within the variational framework described above.

\subsubsection{State manifold}

Since we consider a coupled problem involving diffusion inside a moving domain, we choose the manifold of pairs $(\Omega, c)$ where $\Omega$ is a bounded domain in $\mathbb{R}^{N}$ and $c$ is a non-negative solute concentration such that $\operatorname{supp} c \subset \bar{\Omega}$. Accordingly, its tangent space consists of pairs $\left(V_{n}, c_{t}\right)$ where $V_{n}$ is has the same meaning as above and $c_{t}$ is a scalar function in $\Omega$ representing concentration changes.

\subsubsection{Energy functional}

Keeping in mind the examples above, we define

$$
\mathscr{E}(\Omega, c)=\gamma \int_{\Omega} c \ln c d x+\alpha|\partial \Omega|
$$

with appropriate positive constants $\alpha$ and $\gamma$. This includes diffusion and surface tension as driving mechanisms of the evolution. 


\subsubsection{Process space}

The processes that cause energy dissipation here are solvent motion, solute flux, and passage of the solvent through the membrane. As in the examples above, the former two are described by a velocity field $u$ and a flux field $f$. Since the solvent flux through the membrane is given by $\left.u\right|_{\partial \Omega} \cdot n-V_{n}$ and in view of (2.5) it makes sense to consider $V_{n}$ as a process component as well. We therefore choose (cf. (2.3), (2.5))

$$
\mathscr{P}_{(\Omega, c)}=\left\{\left(u, f, V_{n}\right) \mid \operatorname{div} u=0 \text { in } \Omega, f \cdot n=c V_{n} \text { on } \partial \Omega\right\} .
$$

\subsubsection{Process map}

In view of (2.4) it is now straightforward to define

$$
\Pi_{(\Omega, c)}\left(u, f, V_{n}\right)=\left(V_{n},-\operatorname{div} f\right) .
$$

\subsubsection{Dissipation}

Concerning dissipation by the motion of the solutes, we have to consider now the flux relative to the flux $c u$ arising from pure transport by the solvent. The dissipation by inner friction is the same as described in the example above. Additionally, we model the resistance that solvent particles have to overcome when they cross the membrane. Since we assume a linear constitutive relation here as well, we find

$$
\Psi_{(\Omega, c)}\left(u, f, V_{n}\right):=\frac{\eta_{1}}{2} \int_{\Omega} \frac{|f-c u|^{2}}{c} d x+\frac{\eta_{2}}{2} \int_{\Omega}|\varepsilon(u)|^{2} d x+\frac{\eta_{3}}{2} \int_{\partial \Omega}\left(u \cdot n-V_{n}\right)^{2} d \sigma .
$$

The constants $\eta_{1}$ and $\eta_{2}$ have the same meaning as in the introductory examples above, and $\eta_{3}$ can be interpreted as the inverse permeability of the membrane.

These choices complete the modelling of our problem, in the sense that the model is fully specified by them.

\section{Remark}

(1) Observe that for $c>0$, the normal velocity $V_{n}$ is determined by (2.5). This suggests the alternative (but, in this case, equivalent) choices

$$
\begin{aligned}
& \tilde{\mathscr{P}}_{(\Omega, c)}=\{(u, f) \mid \operatorname{div} u=0 \text { in } \Omega\}, \\
& \tilde{\Pi}_{(\Omega, c)}=\left(\left.\frac{1}{c} f\right|_{\partial \Omega} \cdot n,-\operatorname{div} f\right) .
\end{aligned}
$$

This appears to be more elegant and straightforward because the process space is smaller and the process map is completely dictated by solute mass conservation. However, we refrain from this as the restriction to everywhere strictly positive concentrations is both unnatural and unnecessary in our analysis of the resulting moving boundary problem (More generally, there is a freedom of choice of the process space and map independent of the physics of the problem.) 
(2) If we restrict the manifold of states to any submanifold consisting of pairs $(\Omega, c)$ that satisfy

$$
\int_{\Omega} c d x=M_{0}
$$

with $M_{0}$ being a fixed total solute mass then the process maps $\Pi_{(\Omega, c)}$ are easily seen to be surjective.

(3) In a more sophisticated version, elasticity and bending stiffness of the membrane could be included by choosing energy functionals as in [5]. Likewise, it is possible to include inertia forces which would yield a Navier-Stokes type problem.

We next derive the evolution equations for the state $(\Omega, c)$ under our modelling assumptions and turn to the minimisation problem (2.2). We first observe that

$$
\mathscr{E}^{\prime}(\Omega, c)\left[\Pi_{(\Omega, c)}\left(u, f, V_{n}\right)\right]=\gamma \int_{\Omega} \frac{\nabla c}{c} \cdot f d x-\int_{\partial \Omega}(\alpha H+\gamma c) V_{n} d \sigma .
$$

As usual, we account for the incompressibility condition (2.3) by introducing a Lagrange multiplier $q$, which physically represents the hydrodynamic pressure. Thus the stationarity conditions are the vanishing of the first variation of

$$
L\left(u, f, V_{n}, q\right):=\Psi\left(u, f, V_{n}\right)+\gamma \int_{\Omega} \frac{\nabla c}{c} \cdot f d x-\int_{\partial \Omega}(\alpha H+\gamma c) V_{n} d \sigma-\int_{\Omega} q \operatorname{div} u d x
$$

with respect to all variations $\left(\tilde{u}, \tilde{f}, \tilde{V}_{n}\right)$ that satisfy $\tilde{f} \cdot n=c \tilde{V}_{n}$ on $\partial \Omega$.

Explicitly, this means

$$
\begin{aligned}
\int_{\partial \Omega} & \left(-\eta_{3}\left(u \cdot n-V_{n}\right)-\alpha H-\gamma c\right) \tilde{V}_{n} d \sigma=0 \\
& -\eta_{1} \int_{\Omega}(f-c u) \cdot \tilde{u} d x+\eta_{2} \int_{\Omega} \varepsilon(u): \varepsilon(\tilde{u}) d x-\eta_{3} \int_{\partial \Omega}\left(V_{n}-u \cdot n\right) \tilde{u} \cdot n d \sigma-\int_{\Omega} q \operatorname{div} \tilde{u} d x \\
= & \int_{\Omega}\left(-\frac{\eta_{2}}{2} \Delta u+\nabla q-\eta_{1}(f-c u)\right) \cdot \tilde{u} d x+\int_{\partial \Omega}\left(\tau(u, q) n-\eta_{3}\left(V_{n}-u \cdot n\right) n\right) \cdot \tilde{u} d \sigma=0 \\
& \times \int_{\Omega} \frac{\eta_{1}(f-c u)+\gamma \nabla c}{c} \cdot \tilde{f} d x=0,
\end{aligned}
$$

where

$$
\tau(u, q)=\eta_{2} \varepsilon(u)-q I,
$$

is the (hydrodynamic) stress tensor in the solvent as before.

Gathering all equations and eliminating $f$ by means of $(2.6)_{3}$ we obtain the moving boundary problem

$$
\left.\begin{array}{rlll}
-\frac{\eta_{2}}{2} \Delta u+\nabla(q+\gamma c) & =0 & \text { in } \Omega(t), & t>0, \\
\operatorname{div} u=0 & \text { in } \Omega(t), & t>0, \\
\partial_{t} c-\frac{\gamma}{\eta_{1}} \Delta c+\nabla c \cdot u=0 & \text { in } \Omega(t), & t>0, \\
\tau(u, q+\gamma c) n-\alpha H n=0 & \text { on } \partial \Omega(t), & t>0, \\
\alpha H+\gamma c+\eta_{3}\left(u \cdot n-V_{n}\right) & =0 & \text { on } \partial \Omega(t), & t>0, \\
-\frac{\gamma}{\eta_{1}} \partial_{n} c+c u \cdot n-c V_{n} & =0 & \text { on } \partial \Omega(t), & t>0 .
\end{array}\right\}
$$


To enforce uniqueness of the solution, in our one-phase setting one has to exclude rigid body motions. For this, we shall additionally demand that $u$ is $L^{2}(\Omega)$-orthogonal to the space of rigid body velocities on $\mathbb{R}^{N}$. Of course, the system has to be complemented by initial conditions for $c$ and $\Omega$.

The model proposed here contains a number of more or less well-studied moving boundary problems as (formal) limit cases:

- Osmosis in a resting solvent: When $\eta_{2} \rightarrow \infty$, the solvent becomes immobile, and (2.7) reduces to the problem of the motion of a membrane under the influence of osmosis and surface tension:

$$
\left.\begin{array}{rll}
\partial_{t} c-\frac{\gamma}{\eta_{1}} \Delta c=0 & \text { in } \Omega(t), & t>0, \\
\alpha H+\gamma c-\eta_{3} V_{n}=0 & \text { on } \partial \Omega(t), & t>0, \\
\frac{\gamma}{\eta_{1}} \partial_{n} c+c V_{n}=0 & \text { on } \partial \Omega(t), & t>0 .
\end{array}\right\} .
$$

This model has been discussed in one spatial dimension (under the name "closed osmometer problem") in [9,21], in a radially symmetric setting in [24-26] and in higher dimensions in $[13,14]$, with the latter reference discussing the two-phase setting in terms of stability of equilibria.

- Fast diffusion: When $\eta_{1} \rightarrow 0$, the concentration is forced on the spatially constant value $c=c(t)=M /|\Omega(t)|$, where $M$ is the total mass of solute. To our knowledge, the resulting Stokes problem $(2.7)_{1},(2.7)_{2},(2.7)_{4},(2.7)_{5}$ has not yet attracted any attention. Starting from (2.8), however, one obtains the surface motion law $(2.8)_{2}$, which is just mean curvature flow with a non-local "braking term" [17].

- Impermeable membrane: When $\eta_{3} \rightarrow \infty$, the condition $V_{n}=u \cdot n$ is enforced, i.e. the membrane simply moves according to the normal component of the velocity field. This is the standard kinematic boundary condition for moving liquid surfaces, also in cases without a membrane. So the resulting problem

$$
\left.\begin{array}{rlll}
-\frac{\eta_{2}}{2} \Delta u+\nabla(q+\gamma c) & =0 & \text { in } \Omega(t), & t>0, \\
\operatorname{div} u=0 & \text { in } \Omega(t), & t>0, \\
\partial_{t} c-\frac{\gamma}{\eta_{1}} \Delta c+\nabla c \cdot u=0 & \text { in } \Omega(t), & t>0, \\
\tau(u, q+\gamma c) n-\alpha H n=0 & \text { on } \partial \Omega(t), & t>0, \\
u \cdot n-V_{n}=0 & \text { on } \partial \Omega(t), & t>0, \\
\partial_{n} c=0 & \text { on } \partial \Omega(t), & t>0
\end{array}\right\},
$$

describes the free motion of a drop of viscous liquid under the influence of its own surface tension, combined with a convection-diffusion problem of solute inside the drop. It is interesting to observe that in the impermeable case the presence of a solute does not influence the evolution of the domain: for any smooth evolution $t \mapsto(\Omega(t), c(t))$, the evolution $t \mapsto(\Omega(t), 0)$ satisfies (2.9) as well, i.e. $t \mapsto \Omega(t)$ depends on $\Omega(0)$ only. More precisely, the domain evolution is given by the Stokes flow problem with surface tension described above. The reason for this can be understood in the following terms: While the presence of solute appears in the Stokes equations (and dynamic boundary condition) only via a modified pressure term, it is only the velocity field which determines the domain evolution. 
To normalise all but one of the occurring constants to one we non-dimensionalise the equations using the characteristic quantities

$$
L:=\frac{\tilde{\eta}_{2}}{\eta_{3}}, \quad T:=\frac{L^{2} \eta_{1}}{\gamma}, \quad F:=\frac{L^{N-1} \tilde{\eta}_{2}}{T}, \quad M:=\frac{L F}{\gamma},
$$

$\left(\tilde{\eta}_{2}:=\eta_{2} / 2\right)$ for length, time, force, and molarity, respectively. Keeping the same symbols to denote dimensionless variables, we rewrite (2.7) in the form

$$
\begin{aligned}
& -\Delta u+\nabla(q+c)=0 \quad \text { in } \Omega(t), \quad t>0, \\
& \operatorname{div} u=0 \quad \text { in } \Omega(t), \quad t>0, \\
& \tau(u, q+c) n=\kappa H n \quad \text { on } \partial \Omega(t), t>0, \\
& \partial_{t} c-\Delta c=-\nabla c \cdot u \quad \text { in } \Omega(t), \quad t>0,
\end{aligned}
$$

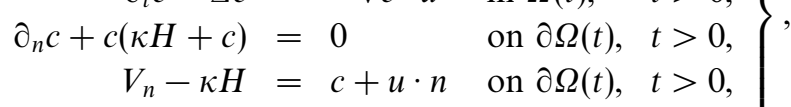

$$
\begin{aligned}
& \Omega(0)=\Omega_{0}, \\
& c(0)=c_{0} \quad \text { in } \Omega_{0} \text {, }
\end{aligned}
$$

where $\tau$ denotes now the mapping $(u, r) \mapsto 2 \varepsilon(u)-r I=\left(\nabla u+(\nabla u)^{T}\right)-r I$ and $\kappa:=\frac{\alpha \eta_{1} \tilde{\eta}_{2}^{N-1}}{\gamma \eta_{3}^{N}}$.

\section{Short-time well-posedness}

\subsection{Preliminaries and formulation of the main result}

If $U \subset \mathbb{R}^{l}(l \in \mathbb{N})$ is an open set and $X$ is a Banach space, let $B U C(U, X)$ be the Banach space of all bounded and uniformly continuous $X$-valued functions on $U$. The space $B U C^{k}(U, X)$ contains those elements of $B U C(U, X)$ that possess bounded and uniformly continuous derivatives up to order $k \in \mathbb{N}$. For $k \in \mathbb{N} \cup\{0\}$ and $s \in(0,1), B U C^{k+s}(U, X)$ denotes the usual $X$-valued Hölder space. The little Hölder space $h^{k+s}(U, X)$ is defined by

$$
\begin{aligned}
& h^{s}(U, X):=\left\{f \in B U C^{s}(U, X) ; \lim _{h \rightarrow 0} \sup _{x, y \in U ;|x-y|<h} \frac{\|f(x)-f(y)\|_{X}}{|x-y|^{s}}=0\right\}, \\
& h^{k+s}(U, X):=\left\{f \in B U C^{k+s}(U, X) ; \partial^{\beta} f \in h^{s}(U, X) ;|\beta|=k\right\} .
\end{aligned}
$$

If $U$ is a domain with sufficiently regular boundary and $k<l \in \mathbb{N}$, then $h^{k+s}(U, X)$ is known to be the closure of $B U C^{l}(U, X)$ in $B U C^{k+s}(U, X)$. Hence, if $l+r>k+s$, $h^{l+r}(U, X)$ is dense in $h^{k+s}(U, X)$ in that case. More basic properties of the little Hölder spaces can be found in [16]. In the case $X=\mathbb{R}$, we write $B U C(U):=B U C(U, \mathbb{R})$, $B U C^{k}(U):=B U C^{k}(U, \mathbb{R})$ etc.

If $X, Y$ are Banach spaces, $\mathscr{L}(X, Y)$ denotes the Banach space of bounded linear mappings from $X$ to $Y$. All spaces are equipped with their natural topologies. As usual, function spaces over a manifold are defined by means of a sufficiently smooth atlas. 
First, we consider the Stokes equations on a general domain in the regularity scale of little Hölder spaces:

Let $\beta \in(0,1)$ and $\Xi$ be a bounded $C^{3+\beta}$ domain in $\mathbb{R}^{N}$ (that is a bounded open set whose boundary possesses a $C^{3+\beta}$-atlas) with exterior unit normal $n=n_{\partial \Xi}$. Let $V_{0}$ be the vector space of rigid body velocities on $\mathbb{R}^{N}$, i.e.

$$
V_{0}:=\left\{v: \mathbb{R}^{N} \rightarrow \mathbb{R}^{N} ; v_{i}(x)=\sum_{j} s_{i j} x_{j}+c_{i} ; s_{i j}, c_{i} \in \mathbb{R} ; s_{i j}=-s_{j i}\right\} .
$$

Fix a basis $\left\{\phi_{k}\right\}$ in $V_{0}$ and define the linear operator $\ell \in \mathscr{L}\left(L^{2}\left(\Xi, \mathbb{R}^{N}\right), V_{0}\right)$ by

$$
\ell(w)=\ell_{\Xi}(w)=\sum_{k}\left(w, \phi_{k}\right) \phi_{k}, \quad\left(w, \phi_{k}\right):=\int_{\Xi} w \cdot \phi_{k} d x .
$$

We introduce the spaces

$$
\mathscr{X}(\Xi):=h^{2+\beta}\left(\Xi, \mathbb{R}^{N}\right) \times h^{1+\beta}(\Xi), \quad \mathscr{Y}(\Xi):=h^{\beta}\left(\Xi, \mathbb{R}^{N}\right) \times h^{1+\beta}(\Xi) \times h^{1+\beta}\left(\partial \Xi, \mathbb{R}^{N}\right)
$$

\section{Lemma 3.1}

(i) The linear operator

$$
\Lambda=\Lambda_{\Xi} \in \mathscr{L}(\mathscr{X}(\Xi), \mathscr{Y}(\Xi))
$$

given by

$$
\Lambda(u, p):=(-\Delta u+\nabla p+\ell(u), \operatorname{div} u, \tau(u, p) n),
$$

is a topological isomorphism.

(ii) If $(u, p)=\Lambda^{-1}(f, g, h)$ and $(f, g, h)$ satisfies the solvability conditions

$$
\int_{\Xi}(f-\nabla g) \cdot \phi d x-\int_{\partial \Xi} h \cdot \phi d S=0 \quad \text { for all } \phi \in V_{0},
$$

then $\ell(u)=0$.

Proof This result is essentially stated (in large Hölder spaces and without proof) in [22], Proposition 2, see also p. 645. A proof of a corresponding result in Sobolev spaces can be found in [10, Lemma 2]. Basically, the proof is based on the fact that the operator given by

$$
(u, p) \mapsto(-\Delta u+\nabla p, \operatorname{div} u, \tau(u, p) n)
$$

describes a Douglis-Nirenberg elliptic boundary system that satisfies the LopatinskiiShapiro condition and is therefore a Fredholm operator from $\mathscr{X}(\Xi)$ to $\mathscr{Y}(\Xi)$. Its kernel is easily seen to be $V_{0} \times\{0\}$, and a discussion of the weak formulation shows that its index is zero and yields the solvability conditions. Introducing the auxiliary term $\ell(u)$ accomplishes the reduction to the case of an isomorphism, cf. [23], Lemma 21.1. 
Fix $0<\beta<\alpha<1$. We assume that

(I1) $\Omega_{0} \subset \mathbb{R}^{N}$ is a domain and $\Gamma_{0}:=\partial \Omega_{0}$ is a closed compact hypersurface of regularity class $h^{4+\beta}$;

(I2) $c_{0} \in h^{2+\alpha}\left(\Omega_{0}\right)$ satisfies $\partial_{n} c_{0}+\kappa H_{\Gamma_{0}} c_{0}+c_{0}^{2}=0$ on $\Gamma_{0}$.

We use the direct mapping method to transform system (2.10) into a set of equations given over a fixed and smooth reference domain. The unknown family of surfaces $\{\Gamma(t)\}:=$ $\{\partial \Omega(t)\}$ will be described by a signed distance function with respect to that surface. In order to carry out these transformations, we need some preparation:

Given any closed compact hypersurface $\Sigma$ of class $C^{2}$, let $T_{\delta}=T_{\delta}(\Sigma)$ be an open tubular neighborhood of $\Sigma$, i.e. the diffeomorphic image of the mapping

$$
X_{\Sigma}: \Sigma \times(-\delta, \delta) \rightarrow \mathbb{R}^{N}, \quad(x, a) \mapsto x+a n_{\Sigma}(x)
$$

where $n_{\Sigma}(x)$ is the outer unit normal vector at $x \in \Sigma$ and $\delta>0$ is sufficiently small. It is convenient to decompose the inverse of $X_{\Sigma}$ into $X_{\Sigma}^{-1}=\left(P_{\Sigma}, \Lambda_{\Sigma}\right)$, where $P_{\Sigma}(x)$ is the metric projection of a point $x \in T_{\delta}$ onto $\Sigma$ and $\Lambda_{\Sigma}$ is the signed distance function with respect to $\Sigma$. Let

$$
\operatorname{Ad}_{\Sigma, \varepsilon}:=\left\{\sigma \in C^{1}(\Sigma) ;\|\sigma\|_{C(\Sigma)}<\varepsilon / 5\right\} \quad(\varepsilon>0)
$$

be the set of admissible boundary perturbations. If $\varepsilon>0$ is small enough, then the mapping $\theta_{\sigma}(x):=x+\sigma(x) n_{\Sigma}(x)$ is for each $\sigma \in \operatorname{Ad}_{\Sigma, \varepsilon}$ a diffeomorphism mapping $\Sigma$ onto $\Sigma_{\sigma}:=\theta_{\sigma}[\Sigma]$.

Due to Theorem 4.2 in [3] we can fix a number $\delta>0$ and a triple $\left(\Omega, S_{\delta}(\Gamma), \rho_{0}\right)$ in the following way:

- $\Omega \subset \Omega_{0}$ is a domain and $\Gamma:=\partial \Omega$ is a closed compact real analytic hypersurface;

- $S:=S_{\delta}(\Gamma)$ is an open tubular neighborhood of $\Gamma, \Gamma_{0} \subset S$;

- $\rho_{0} \in h^{4, \beta}(\Gamma) \cap \operatorname{Ad}_{\Gamma, \delta}$ and the mapping $\theta_{\rho_{0}}: \Gamma \rightarrow \Gamma_{0}$ is a $h^{4, \beta}$ - diffeomorphism. In particular, $\Gamma_{0}=\Gamma_{\rho_{0}}=\theta_{\rho_{0}}[\Gamma]$.

From now on let $\delta>0,\left(\Omega, S, \rho_{0}\right)$ be chosen as described above and let $\mathrm{Ad}:=\operatorname{Ad}_{\Gamma, \delta}$.

Observe that $\theta_{\sigma}[\Gamma] \subset S$ for all $\sigma \in$ Ad. Suppose that $\sigma \in \operatorname{Ad} \cap h^{m+\gamma}(\Gamma)$ for some $(m, \gamma) \in \mathbb{N} \times(0,1)$. It is not difficult to see that then $\theta_{\sigma} \in h^{m+\gamma}\left(\Gamma, \mathbb{R}^{N}\right)$ and $\theta_{\sigma}^{-1} \in h^{m+\gamma}\left(\Gamma_{\sigma}, \mathbb{R}^{N}\right)$. Moreover, given $\sigma \in \operatorname{Ad} \cap h^{m+\gamma}(\Gamma)$, the mapping $\theta_{\sigma}$ extends to a diffeomorphism

$$
\theta_{\sigma} \in \operatorname{Diff}^{m+\gamma}\left(\mathbb{R}^{N}, \mathbb{R}^{N}\right),\left.\quad \theta_{\sigma}\right|_{\Omega} \in \operatorname{Diff}^{m+\gamma}\left(\Omega, \Omega_{\sigma}\right) \quad\left(\Omega_{\sigma}:=\theta_{\sigma}[\Omega]\right),
$$

such that we have $\partial \Omega_{\sigma}=\Gamma_{\sigma}$. The extension (called the Hanzawa-diffeomorphism) is given by

$$
\theta_{\sigma}(y)=\left\{\begin{aligned}
P_{\Gamma}(y)+\left[\Lambda_{\Gamma}(y)+\mu\left(\Lambda_{\Gamma}(y)\right) \sigma\left(P_{\Gamma}(y)\right)\right] \cdot n_{\Gamma}\left(P_{\Gamma}(y)\right), & y \in S \\
y, & y \notin S,
\end{aligned}\right.
$$


where $\mu \in \mathscr{D}(\mathbb{R})$ satisfies $\left.\mu\right|_{[-\delta / 5, \delta / 5]}=1, \operatorname{supp}(\mu) \subset(-(3 \delta) / 5,(3 \delta) / 5),\left|\mu^{\prime}\right| \leqslant 5 / \delta$. Note that, for $\sigma \in \operatorname{Ad}$ the surface $\Gamma_{\sigma}$ is the zero level set of the function $\varphi_{\sigma}$ defined by as follows:

$$
\varphi_{\sigma}(x)=\Lambda_{[\Gamma]}(x)-\sigma\left(P_{[\Gamma]}(x)\right),
$$

$x \in S$, i.e. $\Gamma_{\sigma}=\varphi_{\sigma}^{-1}[\{0\}]$. For later use we set

$$
L_{\sigma}(x):=\left|\nabla \varphi_{\sigma}\right|\left(\theta_{\sigma}(x)\right)
$$

It can be shown that $L_{\sigma}>0$ on $\Gamma$ for all $\sigma \in$ Ad. Finally, if $\rho:[0, T] \rightarrow$ Ad is time dependent, we use the notation

$$
\Omega_{\rho, T}:=\bigcup_{t \in(0, T)}\{t\} \times \Omega_{\rho(t)} \subset \mathbb{R}^{N+1} .
$$

We are now ready to introduce the notion of a classical solution of (2.10):

Definition 3.2 Let $c_{0}, \Gamma_{0}$ satisfy (I1) and (I2), and let $\mathcal{O}:=h^{4, \beta}(\Gamma) \cap$ Ad inherit the topology of $h^{4, \beta}(\Gamma)$. A time-dependent family of domains $\{\Omega(t) ; t \in[0, T]\}$, functions $c(t), q(t): \bar{\Omega}(t) \rightarrow \mathbb{R}$ and a vector field $u(t): \bar{\Omega}(t) \rightarrow \mathbb{R}^{N}$ form a classical solution of (2.10) on $[0, T]$, if there exists a function $\rho \in C([0, T], \mathcal{O}) \cap C^{1}\left([0, T], h^{2, \beta}(\Gamma)\right)$ such that letting $\Gamma(t):=\partial \Omega(t)$

(i) $\Omega(t)=\Omega_{\rho(t)}, t \in[0, T]$ (thus also $\left.\Gamma(t)=\Gamma_{\rho(t)}\right)$;

(ii) $c(\cdot) \circ \theta_{\rho(\cdot)} \in C\left([0, T], h^{2+\alpha}(\Omega)\right) \cap C^{1}\left([0, T], h^{\alpha}(\Omega)\right)$;

(iii) $(u, q)(t) \in h^{3+\beta}\left(\Omega(t), \mathbb{R}^{N}\right) \times h^{2+\beta}(\Omega(t))$ for $t \in[0, T]$;

(iv) $t \mapsto(c(t), u(t), q(t), \Omega(t))$ satisfies the equations of (2.10) pointwise on $[0, T]$, and, additionally, $\ell_{\Omega_{\rho(t)}}(u(t))=0$ for $t \in[0, T]$.

Note that, (ii) in particular implies that $c \in C^{1,2}\left(\Omega_{\rho, T}, \mathbb{R}\right) \cap B U C\left(\Omega_{\rho, T}, \mathbb{R}\right)$ and $c(t) \in$ $h^{2+\alpha}\left(\Omega_{\rho(t)}\right)$ for $t \in[0, T]$. The main theorem of this section reads as follows:

Theorem 3.3 Let $c_{0}, \Gamma_{0}$ satisfy (I1) and (I2). Then there exists a positive time $T$ and a unique classical solution $t \mapsto(c(t), u(t), q(t), \Omega(t))$ of (2.10) on $[0, T]$.

\subsection{Transformation to a fixed interface}

Given $\sigma \in \operatorname{Ad}$, let $\theta_{\sigma}^{*}, \theta_{*}^{\sigma}$ denote the pull-back and push-forward operators induced by $\theta_{\sigma}$, i.e. $\theta_{\sigma}^{*} f=f \circ \theta_{\sigma}, \theta_{*}^{\sigma} g=g \circ \theta_{\sigma}^{-1}$. For functions $b=b(t, x), \rho=\rho(t, x)$ that depend on time we define $\left[\theta_{\rho}^{*} b\right](t, x):=\left[\theta_{\rho(t)}^{*} b(t, \cdot)\right](x)$, analogue for $\theta_{*}^{\rho}$.

First, we consider the transformed Stokes equations. For $\rho \in \operatorname{Ad} \cap h^{3+\beta}(\Gamma)$ observe that (cf. [10, Lemma 1])

$$
\int_{\Gamma_{\rho}} H_{\Gamma_{\rho}} n_{\Gamma_{\rho}} \cdot \phi d S=0 \quad \text { for all } \phi \in V_{0}
$$


Thus, letting

$$
\begin{aligned}
H(\rho) & :=\theta_{\rho}^{*} H_{\Gamma_{\rho}}, \\
n(\rho) & :=\theta_{\rho}^{*} n_{\Gamma_{\rho}},
\end{aligned}
$$

in view of Lemma 3.1 it makes sense to define

$$
s(\rho):=\theta_{\rho}^{*} v,
$$

where $(v, q) \in h^{2+\beta}\left(\Omega_{\rho}, \mathbb{R}^{N}\right) \times h^{1+\beta}\left(\Omega_{\rho}\right)$ is the unique solution of

$$
\left.\begin{array}{rlrl}
-\Delta v+\nabla q & =0 & & \text { in } \Omega_{\rho}, \\
\operatorname{div} v & =0 & & \text { in } \Omega_{\rho}, \\
\tau(v, q) n_{\Gamma_{\rho}} & =\kappa H_{\Gamma_{\rho}} n_{\Gamma_{\rho}} & & \text { on } \Gamma_{\rho}, \\
\ell_{\Omega_{\rho}}(v) & =0 . & &
\end{array}\right\} .
$$

This mapping is smooth:

Lemma 3.4 We have $[\rho \mapsto s(\rho)] \in C^{\infty}\left(\operatorname{Ad} \cap h^{3+\beta}(\Gamma), h^{2+\beta}\left(\Omega, \mathbb{R}^{N}\right)\right)$.

Proof Recall our notation (3.1). For $\rho \in \operatorname{Ad} \cap h^{3+\beta}(\Gamma)$ we have that the pull-back $\theta_{\rho}^{*}$ induces isomorphisms from $\mathscr{X}\left(\Omega_{\rho}\right)$ to $\mathscr{X}(\Omega)$ and from $\mathscr{Y}\left(\Omega_{\rho}\right)$ to $\mathscr{Y}(\Omega)$ which we will denote by the same symbols. The corresponding inverse will be denoted by $\theta_{*}^{\rho}$. Define

$$
\Lambda(\rho):=\theta_{\rho}^{*} \Lambda_{\Omega_{\rho}} \theta_{*}^{\rho},
$$

observe that by Lemma $3.1 \Lambda(\rho) \in \mathscr{L}_{i s}(\mathscr{X}(\Omega), \mathscr{Y}(\Omega))$ and

$$
\left(s(\rho), \theta_{\rho}^{*} q\right)=\Lambda(\rho)^{-1}(0,0, \kappa H(\rho) n(\rho)),
$$

with $q$ from (3.2). As

$$
[\rho \mapsto \kappa H(\rho) n(\rho)] \in C^{\infty}\left(\operatorname{Ad} \cap h^{3+\beta}(\Gamma), h^{1+\beta}\left(\Omega, \mathbb{R}^{N}\right)\right),
$$

it remains to show that

$$
[\rho \mapsto \Lambda(\rho)] \in C^{\infty}\left(\operatorname{Ad} \cap h^{3+\beta}(\Gamma), \mathscr{L}(\mathscr{X}(\Omega), \mathscr{Y}(\Omega)) .\right.
$$

The result follows then from the fact that taking the inverse of an isomorphism is a smooth operation. To show (3.3) it is sufficient to explicitly carry out the transformations of the differential and integral operators involved. For example, for a first-order partial derivative $\partial_{i}$ we have

$$
\theta_{\rho}^{*} \partial_{i} \theta_{*}^{\rho}=a_{i}^{j} \partial_{j}
$$

where $a_{i}^{j}=a_{i}^{j}(\rho) \in h^{2+\beta}(\bar{\Omega})$ is an element of the matrix $\left(D \theta_{\rho}\right)^{-1}$ and therefore depends smoothly on $\rho \in \operatorname{Ad} \cap h^{3+\beta}(\Gamma)$. Similarly,

$$
\theta_{\rho}^{*}\left(\ell_{\Omega_{\rho}}\left(\theta_{*}^{\rho} v\right)\right)=\sum_{i} \int_{\Omega} v \cdot\left(\psi_{i} \circ \theta_{\rho}\right) \operatorname{det} D \theta_{\rho} d x\left(\psi_{i} \circ \theta_{\rho}\right),
$$

which is smooth in $\rho$ as well. 
In order to transform (2.10) to $\Omega$ for suitable $\rho$ we introduce the operators $\mathscr{A}(\rho), \mathscr{B}(\rho)$, $\mathscr{K}(\rho)$ by

$$
\begin{aligned}
\mathscr{A}(\rho) \xi & :=\theta_{\rho}^{*}\left(\Delta\left(\theta_{*}^{\rho} \xi\right)\right) \\
\mathscr{B}(\rho) \xi & :=\theta_{\rho}^{*}\left(\nabla\left(\theta_{*}^{\rho} \xi\right)\right) \cdot n(\rho) ; \\
\mathscr{K}(\rho) \xi & :=\theta_{\rho}^{*}\left(\nabla\left(\theta_{*}^{\rho} \xi\right)\right) .
\end{aligned}
$$

The transformed problem reads then

$$
\left.\begin{array}{rlrl}
\partial_{t} \xi-\mathscr{A}(\rho) \xi & =R(\xi, \rho)-\mathscr{K}(\rho) \xi \cdot s(\rho) & & \text { in } \Omega, \\
\mathscr{B}(\rho) \xi & =-\kappa \xi H(\rho)-\xi^{2} & & \text { on } \Gamma, \\
\partial_{t} \rho-\kappa L_{\rho} H(\rho) & =L_{\rho}(\xi+s(\rho) \cdot n(\rho)) & & \text { on } \Gamma, \\
\xi(0) & =\xi_{0} & & \\
\rho(0) & =\rho_{0}, &
\end{array}\right\}
$$

where $\xi_{0}:=\theta_{\rho_{0}}^{*} c_{0}$. The term $R$ arises from the transformation of the time derivative and is determined by

$$
R(z, \sigma)(y)=r_{0}\left(L_{\sigma}[\kappa H(\sigma)+z+s(\sigma) \cdot n(\sigma)], B_{\mu}(\sigma) z\right)(y), \quad y \in \Omega,
$$

where $z \in C^{1}(\bar{\Omega}), \sigma \in \operatorname{Ad} \cap C^{2}(\Gamma)$ and

$$
\begin{gathered}
r_{0}(h, k)(y):= \begin{cases}\chi\left(\Lambda_{\Gamma}(y)\right) \cdot h\left(P_{\Gamma}(y)\right) \cdot k(y), & \text { if } y \in \Omega \cap S \\
0, & \text { if } y \in \Omega \backslash(\Omega \cap S),\end{cases} \\
B_{\mu}(\sigma) z(y)=\theta_{\sigma}^{*} \nabla\left(\theta_{*}^{\sigma} z\right)(y) \cdot\left(n_{\Gamma} \circ P_{\Gamma}\right)(y), \quad y \in S
\end{gathered}
$$

$(\chi$ being a suitable cut-off function, $\mathrm{cf}$. $[6,12])$. The derivation of $(3.4)$ is a straightforward calculation $[6,12]$.

Note: If $(\xi, \rho)$ is a sufficiently regular solution of (3.4), then $\left(\theta_{*}^{\rho} \xi, \theta_{*}^{\rho} s(\rho), q, \Gamma_{\rho}\right)$ is a classical solution of (2.10), where $q:=\theta_{*}^{\rho}\left(P_{2} \Lambda(\rho)^{-1}(0,0, \kappa H(\rho) n(\rho))-\xi\right)\left(P_{2}\right.$ denoting the projection on the second component).

It turns out that the local well-posedness of system (3.4) can be proved almost in the same fashion as outlined in Sections 2 and 3 in [13], so we recall the abstract setting from there: If $T>0$ is given and $J_{T}:=[0, T]$, let

$$
\begin{aligned}
E_{0} & :=h^{\alpha}(\Omega) \times h^{2+\beta}(\Gamma), \\
E_{1} & :=h^{2+\alpha}(\Omega) \times h^{4+\beta}(\Gamma), \\
\mathbb{E}_{0}\left(J_{T}\right) & :=B U C\left(J_{T}, E_{0}\right), \\
\mathbb{E}_{1}\left(J_{T}\right) & :=B U C^{1}\left(J_{T}, E_{0}\right) \cap B U C\left(J_{T}, E_{1}\right), \\
\mathbb{F}\left(J_{T}\right) & :=B U C\left(J_{T}, h^{1+\alpha}(\Gamma)\right) \cap h^{(1+\alpha) / 2}\left(J_{T}, C(\Gamma)\right) .
\end{aligned}
$$

To economise notation we drop the $T$ - dependence, i.e. write $\mathbb{E}_{1}$ instead of $\mathbb{E}_{1}\left(J_{T}\right)$ etc. and define the sets

$$
\widetilde{\mathrm{Ad}}=\left\{(v, \psi) \in E_{1} \mid \psi \in \mathrm{Ad}\right\}, \quad \widehat{\mathrm{Ad}}=\left\{w \in \mathbb{E}_{1} \mid w(t) \in \widetilde{\mathrm{Ad}}, t \in[0, T]\right\}
$$

which are open subsets of $E_{1}$ and $\mathbb{E}_{1}$, respectively. Our goal is to write system (3.4) as a 
single operator equation. For this we recall the splitting

$$
H(\rho)=P(\rho) \rho+Q(\rho),
$$

as described for example in [8]. More precisely, $P(\rho)$ can be chosen to be a second order uniformly elliptic operator acting as an isomorphism in various scales of function spaces and depending smoothly on $\rho$. The mapping $Q$ contains only lower order terms. Precise mapping properties of $P(\cdot)$ and $Q$ are given for example in $[6,8,12]$.

Remark 1 To clarify the structure of the non-linear operator $H(\rho)$ let $\Delta_{\rho}$ be the LaplaceBeltrami operator of $\Gamma_{\rho}$ w.r.t the metric inherited from the ambient space $\mathbb{R}^{N}$. Let further $n_{\rho}$ be the outer unit normal field and $H_{\rho}$ be the mean curvature of $\Gamma_{\rho}$. Recall that

$$
H_{\rho}=\left(\Delta_{\rho} \xi_{\rho} \mid n_{\rho}\right)_{\mathbb{R}^{N}}
$$

where $\xi_{\rho}$ assigns to each point of $\Gamma_{\rho}$ its cartesian coordinates and $\Delta_{\rho}$ acts componentwise on $\xi_{\rho}$. Parameterising $\Gamma_{\rho}$ over $\Gamma$ (i.e. letting $\xi_{\rho}(x):=x+\rho(x) \cdot n_{\Gamma}(x), x \in \Gamma$ ) we obtain in local coordinates on $\Gamma$

$$
\Delta_{\rho} \xi_{\rho}=\frac{1}{\sqrt{G_{\rho}}} \sum_{i, j=1}^{N-1} \partial_{i}\left(\sqrt{G_{\rho}} g_{\rho}^{i j} \partial_{j} \xi_{\rho}\right),
$$

where $g_{i j}^{\rho}=\left(\partial_{i} \xi_{\rho} \mid \partial_{j} \xi_{\rho}\right)_{\mathbb{R}^{N}},\left(g_{\rho}^{i j}\right)=\left(g_{i j}^{\rho}\right)^{-1}$ and $G_{\rho}=\operatorname{det}\left(g_{i j}^{\rho}\right)$. From equation (3.7) it is clear that $H(\rho)$ is of second order in $\rho$ and that it has a quasilinear structure.

Let

$$
\begin{aligned}
\mathbb{A}(w)(t) & =\left(\begin{array}{cc}
\mathscr{A}(\rho(t)) & 0 \\
0 & \kappa L_{\rho(t)} P(\rho(t))
\end{array}\right), \\
\tilde{\mathbb{B}}(v, \psi)(\zeta, \chi) & =\mathscr{B}(\psi) \zeta, \\
(\mathbb{B}(w)(v, \sigma))(t) & =\tilde{\mathbb{B}}(w(t))(v(t), \sigma(t)), \\
\mathbb{L}(w) & =\left(\partial_{t}-\mathbb{A}(w), \mathbb{B}(w), \gamma_{t}\right),
\end{aligned}
$$

where $w=(\xi, \rho) \in \widehat{\operatorname{Ad}},(v, \sigma) \in \mathbb{E}_{1},(v, \psi) \in \widetilde{\mathrm{Ad}},(\zeta, \chi) \in E_{1}$ and $\gamma_{t} \in \mathscr{L}\left(\mathbb{E}_{1}, E_{1}\right)$ denotes the time trace map $w \mapsto w(0)$. We have

$$
\begin{aligned}
\mathbb{A} & \in C^{\infty}\left(\widehat{\mathrm{Ad}}, \mathscr{L}\left(\mathbb{E}_{1}, \mathbb{E}_{0}\right)\right), \\
\tilde{\mathbb{B}} & \in C^{\infty}\left(\widehat{\mathrm{Ad}}, \mathscr{L}\left(E_{1}, h^{1+\alpha}(\Gamma)\right)\right), \\
\mathbb{B} & \in C^{\infty}\left(\widehat{\mathrm{Ad}}, \mathscr{L}\left(\mathbb{E}_{1}, \mathbb{F}\right)\right), \\
\mathbb{L} & \in C^{\infty}\left(\widehat{\mathrm{Ad}}, \mathscr{L}\left(\mathbb{E}_{1}, \mathbb{E}_{0} \times \mathbb{F} \times E_{1}\right)\right),
\end{aligned}
$$

cf. [13]. Let $w_{0}=\left(\xi_{0}, \rho_{0}\right)$. For given, fixed $M>\left\|w_{0}\right\|_{E_{1}}$ we define the closed set

$$
\mathscr{C}=\mathscr{C}(M, T):=\left\{w \in \mathbb{E}_{1} \mid, w(0)=w_{0},\|w\|_{\mathbb{E}_{1}} \leqslant M\right\},
$$

and introduce the subspace $\mathbb{Z} \subset \mathbb{E}_{0} \times \mathbb{F} \times E_{1}$ by

$$
\mathbb{Z}=\left\{(f, g, h) \in \mathbb{E}_{0} \times \mathbb{F} \times E_{1} \mid \gamma_{t} g=\tilde{\mathbb{B}}\left(w_{0}\right) h\right\} .
$$


The following lemma collects some facts shown in [13] (Lemmas 3.1-3.5). The symbol $\mathscr{L}_{\text {is }}$ stands for the set of topological isomorphisms.

Lemma 3.5 Let $M>\left\|w_{0}\right\|_{E_{1}}$. There is a $T^{*}=T^{*}\left(M, w_{0}\right)$ and a $C=C\left(w_{0}\right)$ such that if $T \in\left(0, T^{*}\right]$ then $\mathscr{C} \subset \widehat{A d}, \mathbb{L}(\mathscr{C}) \subset \mathscr{L}_{\text {is }}\left(\mathbb{E}_{1}, \mathbb{Z}\right)$ and

$$
\left\|\mathbb{L}(w)^{-1}\right\|_{\mathscr{L}\left(\mathbb{Z}, \mathbb{E}_{1}\right)} \leqslant C, \quad w \in \mathscr{C} .
$$

Thus, our problem can be reformulated as

$$
\mathbb{L}(w) w=F(w):=\left(\mathscr{R}(w), \mathscr{G}(w), w_{0}\right), \quad w \in \mathscr{C},
$$

where

$$
\begin{aligned}
& \mathscr{R}(w)(t)=\left(\begin{array}{c}
R(w(t))-\mathscr{K}(\rho(t)) \xi(t) \cdot s(\rho(t)) \\
L_{\rho(t)}(\kappa Q(\rho(t))+\xi(t)+s(\rho(t)) \cdot n(\rho(t)))
\end{array}\right), \\
& \mathscr{G}(w)(t)=-\kappa \xi(t) H(\rho(t))-\xi(t)^{2}
\end{aligned}
$$

$w=(\xi, \rho)$. In view of Lemma 3.4 it is easily checked that

$$
F \in C^{\infty}\left(\widehat{\mathrm{Ad}}, \mathbb{E}_{0} \times \mathbb{F} \times E_{1}\right),
$$

cf. [13].

Lemma 3.6 (Quasilinear character) Let $\varepsilon>0$ and $M>\left\|w_{0}\right\|_{E_{1}}$ be given. There is a $T^{*}=$ $T^{*}\left(\varepsilon, M, w_{0}\right)$ such that if $T \in\left(0, T^{*}\right], w_{1}, w_{2} \in \mathscr{C}$, then

$$
\begin{aligned}
\left\|\mathbb{L}\left(w_{1}\right)-\mathbb{L}\left(w_{2}\right)\right\|_{\mathscr{L}\left(\mathbb{E}_{1}, \mathbb{Z}\right)} & \leqslant \varepsilon\left\|w_{1}-w_{2}\right\|_{\mathbb{E}_{1}} ; \\
\left\|F\left(w_{1}\right)-F\left(w_{2}\right)\right\|_{\mathbb{Z}} & \leqslant \varepsilon\left\|w_{1}-w_{2}\right\|_{\mathbb{E}_{1}} .
\end{aligned}
$$

Proof The estimate (3.9) has been proven in [13]. Using Lemma 3.4 and the facts that

$$
\begin{aligned}
& \mathscr{K} \in C^{\infty}\left(\operatorname{Ad} \cap h^{3+\beta}(\Gamma), \mathscr{L}\left(h^{1+\alpha}(\Omega), h^{\alpha}\left(\Omega, \mathbb{R}^{N}\right)\right)\right), \\
& R \in C^{\infty}\left(\operatorname{Ad} \cap h^{3+\beta}(\Gamma) \times h^{1+\alpha}(\Omega), h^{\alpha}(\Omega)\right), \\
& n \in C^{\infty}\left(\operatorname{Ad} \cap h^{3+\beta}(\Gamma), h^{2+\beta}\left(\Gamma, \mathbb{R}^{N}\right)\right)
\end{aligned}
$$

the estimate (3.10) results analogously to A.17 and A.18 in [13]. Observe in this connection that pointwise scalar multiplication canonically induces a bounded and bilinear mapping

$$
h^{m+\gamma}\left(M, \mathbb{R}^{l}\right) \times h^{\tilde{m}+\tilde{\gamma}}\left(M, \mathbb{R}^{l}\right) \rightarrow h^{m+\gamma}(M, \mathbb{R}), \quad M \in\{\Gamma, \Omega\},
$$

where $m, \tilde{m}, l \in \mathbb{N} \cup\{0\}, l \geqslant 1, \gamma, \tilde{\gamma} \in(0,1)$ and $\tilde{m}+\tilde{\gamma} \geqslant m+\gamma$.

Theorem 3.7 (Short-time well-posedness) Let $w_{0}=\left(\xi_{0}, \rho_{0}\right) \in h^{2+\alpha}(\bar{\Omega}) \times\left(h^{4+\beta}(\Gamma) \cap A d\right)$ be as specified above. Then there are constants $M, T^{*}>0$ such that (3.8), or, equivalently, (3.4) has precisely one solution in $\mathscr{C}$ for any $T \in\left(0, T^{*}\right]$. 
Proof Observe that $\left(\xi_{0}, \rho_{0}\right)$ satisfy the compatibility condition

$$
\mathscr{B}\left(\rho_{0}\right) \xi_{0}=-\kappa \xi_{0} H\left(\rho_{0}\right)-\xi_{0}^{2}
$$

because of (I2). Moreover, due to Lemma 3.5 we can rewrite (3.8) as a fixed point equation

$$
w=\Phi(w):=\mathbb{L}(w)^{-1} F(w), \quad w \in \mathscr{C} .
$$

Thus, the assertion is an immediate consequence of Lemmas 3.5, 3.6 and the obvious modifications of Lemmas 3.5, 3.6 in [13].

The statement of Theorem 3.3 is a direct consequence of Theorem 3.7 and the observation that for a $C^{4+\beta}$ domain $\Xi$ we have

$$
\Lambda_{\Xi} \in \mathscr{L}_{\text {is }}\left(h^{3+\beta}\left(\Xi, \mathbb{R}^{N}\right) \times h^{2+\beta}(\Xi), h^{1+\beta}\left(\Xi, \mathbb{R}^{N}\right) \times h^{2+\beta}(\Xi) \times h^{2+\beta}\left(\partial \Xi, \mathbb{R}^{N}\right)\right)
$$

\section{Conclusion}

Our modelling approach consisted essentially in "adding up building blocks" from problems with a well-known variational structure, namely, diffusion and Stokes flow with surface tension, for the free energy functional as well as for the dissipation. Even though our evolution can be interpreted as a gradient flow, the character and structural properties of the resulting non-linear problem are (to us) not a priori obvious. In particular, even with the same state space and dissipation functional, different energy functionals may lead to both parabolic and hyperbolic evolutions. An example for this is given by the space of probability measures on the real axis with the Wasserstein metric, where, as is well-known by now, the (generalised) gradient flow with respect to the entropy functional is the heat flow, while a certain class of autocorrelation functionals gives rise to a nonlocal hyperbolic evolution related to Burger's equation [4]. In this respect, the challenging problem arises to find direct connections between structural conditions on the energy and dissipation functionals on one hand and the type or other properties of the corresponding evolution on the other. At the moment, we feel unable to even give reasonably general non-trivial conjectures on this.

It turns out that in our case the resulting evolution is parabolic in the following sense: The associated linear homogeneous evolution is described by an analytic semigroup of operators. In turn, the theory of these semigroups provides the means to prove optimal regularity results for the corresponding linear, non-homogeneous evolution equations that arise from linearising the original problem. For a more precise discussion of this, we refer to [1] or [15].

In our problem, we have to consider a coupled evolution for a pair of functions, one of them given inside the reference domain (with boundary conditions) and the other on its boundary. The generator of the corresponding semigroups is diagonal in highest order, so that known results on the "components" can be applied, including a crucial optimal regularity result (Theorem 1.4) from [15]. Technically, this is the basis for Lemma 3.5 in the present paper. 
Furthermore, it is important for our analysis that the non-local solution operator of the Stokes equations only enters in a lower order term (since the pseudodifferential operator mapping the Neumann normal stress boundary data to the Dirichlet boundary data is of order -1 , cf. Lemma 3.4), and therefore does not occur in the leading linear operator $\mathbb{L}\left(w_{0}\right)$ defined in Section 3.2 .

\section{Acknowledgements}

The authors are indebted to M.M. Zaal for interesting discussions. The first author was supported by DFG SPP 1506 "Transport processes at fluidic interfaces".

\section{References}

[1] Amann, H. (1995) Linear and Quasilinear Parabolic Problems, Birkhäuser, Basel, xxxvi+335 pp.

[2] AntanovskiI, L. K. (1993) Analyticity of a free boundary in plane quasi-steady flow of a liquid form subject to variable surface tension. In: Proc. of Conference: The Navier-Stokes Equations II: Theory and Numerical Methods, Oberwolfach 1991, Berlin: Springer, pp. 1-16.

[3] Bergner, M., Escher, J. \& Lippoth, F. (2012) On the blow up scenario for a class of parabolic moving boundary problems. Nonlinear Anal.: T,M\&A 75, 3951-3963.

[4] Bonaschi, G., Carrillo, J., Di Francesco, M. \& Peletier, M. Equivalence of gradient flows and entropy solutions for singular nonlocal interaction equations in 1D. submitted, arXiv: 1310.411.

[5] Cheng, C. H. A, Coutand, D. \& Shkoller, S. (2007) Navier-Stokes equations interacting with a nonlinear elastic biofluid shell. SIAM J. Math. Anal. 39, 742-800.

[6] Escher, J. (2004) Classical solutions for an elliptic parabolic system. Interfaces Free Boundaries 6, 175-193.

[7] Escher, J. \& Prokert, G. (2006) Analyticity of solutions to nonlinear parabolic equations on manifolds and an application to Stokes flow. J. Math. Fluid Mech. 8, 1-35.

[8] Escher, J. \& Simonett, G. (1997) Classical solutions for Hele-Shaw models with surface tension. Adv. Differ. Equ. 2, 619-642.

[9] Frischmuth, K. \& HäNler, M. (1999) Numerical analysis of the closed osmometer problem. Z. Angew. Math. Mech. 79, 107-116.

[10] Günther, M. \& Prokert, G. (1997) Existence results for the quasistationary motion of a capillary liquid drop. Z. Anal. ihre Anwendungen 16, 311-348.

[11] Hopper, R.W. (1990) Plane stokes flow driven by capillarity on a free surface. J. Fluid Mech. 213, 349-375.

[12] KNeISEL, C. (2008) Über das Stefan-Problem mit Oberflächenspannung und thermischer Unterkühlung. PhD Thesis, Universität Hannover 2007; VDM Verlag Dr. Müller.

[13] Lippoth, F. \& Prokert, G. (2012) Classical solutions for a one phase osmosis model. J. Evol. Equ. 12(2), 413-434.

[14] Lippoth, F. \& Prokert, G. (2014) Stability of equilibria for a two-phase osmosis model. NoDEA Nonlinear Differ. Equ. Appl. 21, 129-149.

[15] LunARDI, A. (1989) Maximal space regularity in inhomogeneous initial boundary value parabolic problems. Num. Funct. Anal. Opt. 10(3 and 4), 323-349.

[16] Lunardi, A. (1995) Analytic Semigroups and Optimal Regularity in Parabolic Problems, Birkhäuser, Basel, xviii+424 pp.

[17] Meurs, P. J. P. V. (2011) Osmotic Cell Swelling in the Fast Diffusion Limit. MSc Thesis, Eindhoven University of Technology, http://alexandria.tue.nl/extra1/ afstversl/wsk-i/meurs2011.pdf 
[18] Mielke, A. (2005) Evolution in rate-independent systems. In: Handbook of Differential Equations: Evolutionary Differential Equations, Amsterdam: North-Holland, pp. 461-559.

[19] Peletier, M. A. (2014) Variational Modelling: Energies, Gradient Flows, and Large Deviations, arxiv:1402.1990.

[20] Rayleigh, L. (1913) On the motion of a viscous fluid. London, Edinburgh Dublin Phil. Mag. J. Sci. 26, 776-786.

[21] Rubinstein, L. \& Martuzans, B. (1995) Free Boundary Problems Related to Osmotic Mass Transfer Through Semipermeable Membranes, Gakkotosho, Tokyo, vi+205 pp.

[22] Solonnikov, V. A. (1999) On quasistationary approximation in the problem of motion of a capillary drop. In: J. Escher \& G. Simonett (editors), Topics in Nonlinear Analysis, Birkhäuser, Basel, Progress in Nonlinear Differential Equations and their Applications 35, pp. 643-671.

[23] Vainberg, M. M. \& Trenogin, V. A. (1974) Theory of Branching of Solutions of Non-linear Equations, Noordhoff, Leyden, xxvi+485 pp.

[24] ZaAl, M. M. (2008) Linear Stability of Osmotic Cell Swelling. MSc Thesis, Vrije Universiteit Amsterdam. http://www.few.vu.nl/ mzl400/bin/scriptie.pdf

[25] ZAAL, M. M. (2012) Cell swelling by osmosis: A variational approach. Interfaces Free Boundaries 14, 487-520.

[26] ZaAl, M. M. (2013) Variational Modeling of Parabolic Free Boundary Problems. $\mathrm{PhD}$ Thesis, Vrije Universiteit Amsterdam. http://dare.ubvu.vu.nl/bitstream/ 1871/40209/1/dissertation.pdf 